\title{
Internet écl@ire
}

\author{
@@@France@@@ \\ @@ASN@@
}

@1. Exposition médicale aux rayonnements ionisants : une démarche pluridisciplinaire pour sécuriser le parcours du patient

http://www.asn.fr/index.php/S-informer/Actualites/2013/Exposition-medicale-aux-

rayonnements-ionisants

@ 2. Agréments d'organismes

http://www.asn.fr/index.php/Les-actions-de-l-ASN/La-reglementation/Bulletin-officiel-de-

l-ASN/Agrements-d-organismes

\section{@@@ Europe @@@}

@ 3. Alpha-Immunotherapy: better measurements to improve cancer treatment (JRC Joint Reseach Centre)

http://ec.europa.eu/dgs/jrc/index.cfm?id=1410\&dt_code $=N W S \& o b j \_i d=16460 \& o r i=R S S$

@ 4. Report Human Radiosensitivity - RCE 21(HPA - Health Protection Agency)

This report reviews evidence for variation in human radiosensitivity from epidemiological, clinical and experimental studies, considers mechanisms of radiosensitivity and ethical implications of current and potential future knowledge on the range of radiosensitivity in the human population.

http://www.hpa.org.uk/webw/HPAweb\&HPAwebStandard/HPAweb_C/1317138381727

@5. Prise de position suisse relative aux expositions radiologiques non-médicales (OFSP - Office fédéral de la santé publique)

http://www.bag.admin.ch/ksr-

cpr/04309/04310/index.html?lang $=$ fr\&download $=N H z L p Z e g 7 t$, lnp6IONTU042l2Z6ln1ae 2

IZn4Z2qZpnO2Yuq2Z6gpJCLdH9,fWym162epYbg2c_JjKbNoKSn6A--

@@@Grandes agences@@@

@6. Rapport mondial sur les risques pour la santé de l'accident nucléaire de Fukushima (WHO - World Health Organization)

http://www.who.int/mediacentre/news/releases/2013/fukushima_report_20130228/fr/index .html

Pour télécharger le rapport (en anglais) :

http://www.who.int/ionizing_radiation/pub_meet/fukushima_risk_assessment_2013/en/

DOI: $10.1051 /$ radiopro/2013068

RADIOPROTECTION - VOL. 48 - Nº 2 (2013) 
INTERNET ÉCL@IRE

@@@ À consulter également @ @ @

@ 7. Fukushima Radiation Proves Less Deadly Than Feared (Bloomberg)

Dans cet article, les médecins Robert Peter Gale et Eric Lax font part de leurs observations et de leurs réflexions sur les conséquences de l'accident nucléaire de Fukushima Daiichi.

http://www.bloomberg.com/news/print/2013-03-10/fukushima-radiation-proves-lessdeadly-than-feared.html

@8. La Grande-Bretagne devient partenaire du RJH (Réacteur Jules Horowitz) (CEA) http://www.cea.fr/le-cea/actualites/la-grande-bretagne-devient-partenaire-du-rjh-re106848

\section{@@@Rayonnements non-ionisants @@@}

@9. Exposition des travailleurs aux risques dus aux champs électromagnétiques : guide d'évaluation des risques (INRS - Institut National de Recherche et de Sécurité)

http://www.inrs.fr/accueil/produits/mediatheque/doc/publications.html?refINRS=ED\%206 136

[Dernières consultations des pages web le 2 avril 2013]. Sandrine Figueiredo

IRSN 